\title{
Fiberoptic Bronchoscopy in Neonatal and Pediatric Intensive Care Units: A 5-Year Experience
}

\author{
Lan Fang Tang Zhi Min Chen \\ Department of Pediatric Pulmonology, The Children's Hospital of Zhejiang University School of Medicine, \\ Hangzhou, China
}

\section{Key Words}

Fiberoptic bronchoscopy $\cdot$ Intensive care unit $\cdot$ Respiratory disease $\cdot$ Neonate $\cdot$ Child

\begin{abstract}
Objectives: To determine the value and safety of fiberoptic bronchoscopy in neonatal and pediatric intensive care units (NICUs, PICUs). Subject and Methods: A total of 53 fiberoptic bronchoscopy procedures on 47 patients were reviewed. Bronchoalveolar lavage (BAL) was performed in 23 patients. Results: The primary diseases were pneumonia $(n=16)$, foreign body aspiration $(n=14)$, congenital airway abnormality $(n=12)$, trauma and/or following operations $(n=4)$ and Guillain-Barré syndrome $(n=1)$. The major bronchoscopic findings included inflammation in 26 patients, foreign body in 14, congenital airway abnormality in 12 and blood clotting in 3. Microbiology on BAL fluid was positive in 19 of 23 patients. In 23 patients with atelectasis, full and partial re-expansion was obtained in 14 and 6 patients, respectively, at $24 \mathrm{~h}$ after the procedures. The clinical features of 9 patients with sputum retention or blood clotting improved significantly after BAL. Positive or negative microbiologic BAL fluid results changed treatment in 11 patients, leading to marked clinical improvement in 9 patients. Moreover, 13 patients were extubated within $24 \mathrm{~h}$ of bronchoscopy. Conclusion: These data show that fiberoptic bronchoscopy is safe and effective in the diagnosis and therapy of pulmonary disorders in NICUs and PICUs.
\end{abstract}

Copyright $\odot 2009$ S. Karger AG, Basel
(C) 2009 S. Karger AG, Basel

1011-7571/09/0184-0305\$26.00/0

Fax +4161306 1234

E-Mail karger@karger.ch

www.karger.com
Accessible online at: www.karger.com/mpp

\section{Introduction}

Pulmonary disorders are the predominant problem in neonatal and pediatric intensive care units (NICUs, PICUs) [1-3]. Accurate and safe diagnosis as well as therapy is needed at the bedside in this population. The diagnosis and therapeutic utility of fiberoptic bronchoscopy, coupled with its minimal morbidity and mortality, have led to its increasing use in the care of the critically ill patient. Bronchoscopy allows direct inspection of the upper and lower airways, and facilitates the diagnosis and management of a variety of pulmonary disorders. Over the years, the number of these procedures in adult ICUs has greatly increased, with both diagnostic and therapeutic objectives, such as evaluation for airway foreign body aspiration, difficult intubation, management of atelectasis and hemoptysis, diagnosis of nosocomial pneumonia in ventilated patients, and detection of airway lesions [4-8]. However, up to now, there is limited information about the safety and efficacy of bronchoscopy in NICUs and PICUs [9-11]. We reviewed 53 procedures of fiberoptic bronchoscopy in 47 pediatric patients over a period of 5 years to investigate the value and safety of fiberoptic bronchoscopy in the diagnosis and treatment of patients in NICUs and PICUs. 
Table 1. Characteristics and primary disease of 47 patients

\begin{tabular}{lc}
\hline & Patients \\
\hline Male & $33(70.2 \%)$ \\
Female & $14(29.8 \%)$ \\
Male:female ratio & $2.4: 1$ \\
Age & \\
$\quad<1$ month & $3(6.4 \%)$ \\
$\quad \leq 1$ year & $15(31.9 \%)$ \\
$\quad \leq 3$ years & $19(40.4 \%)$ \\
$>3$ years & $10(21.3 \%)$ \\
Primary disease & \\
$\quad$ Pneumonia & $16(34.1 \%)$ \\
$\quad$ Airway foreign body & $14(29.8 \%)$ \\
Congenital abnormality & $12(25.5 \%)$ \\
Trauma and/or following operations & $4(8.5 \%)$ \\
$\quad$ Guillain-Barré syndrome & $1(2.1 \%)$ \\
\hline
\end{tabular}

\section{Subjects and Methods}

Subjects

A total of 47 patients who underwent fiberoptic bronchoscopy in our NICU and PICU during a 5-year period were enrolled in this study. In total, 53 fiberoptic bronchoscopy procedures were performed in 47 patients. Patient characteristics, including age and gender, preoperative presentations, duration of symptoms, route of bronchoscope insertion, bronchoscopic and bronchoalveolar lavage (BAL) findings, complications and prognosis were reviewed. Informed consent was obtained from the patients' parents, and the study was approved by the Ethical Committee, Zhejiang University School of Medicine, Hangzhou, China.

\section{Bronchoscopy}

Atropine $(0.03 \mathrm{mg} / \mathrm{kg})$ and diazepam $(0.1-0.3 \mathrm{mg} / \mathrm{kg})$ were injected intravenously to 24 patients $30 \mathrm{~min}$ before bronchoscopy (BF-3C30, BF-XP40, BF-P40, Olympus Company) [12]. Twentythree patients with mechanical ventilation did not receive atropine and diazepam. Topical anesthesia with $2 \%$ lidocaine was administered.

BAL with normal saline (weight $<20 \mathrm{~kg}: 1 \mathrm{ml} \cdot \mathrm{kg}^{-1}$ each time, 3 times; weight $>20 \mathrm{~kg}$ : $20 \mathrm{ml}$ each time, 3 times) was conducted in 23 patients with -25 to about $-100 \mathrm{~mm} \mathrm{Hg}(-3.3$ to $-13.3 \mathrm{kPa})$ suction. Microbiological analysis of BAL fluid, including bacterial culture and smear analysis, Chlamydia and Mycoplasma pneumonia DNA detection (real-time PCR), immunofluorescence for adenovirus, respiratory syncytial virus, influenza virus, and parainfluenza virus, was performed in 19 patients.

Oxygen was inhaled and electrocardiogram, respiration and arterial oxygen saturation $\left(\mathrm{SaO}_{2}\right)$ were continuously monitored during the procedure to keep $\mathrm{SaO}_{2}$ over $85 \%$. The bronchoscope was withdrawn if the $\mathrm{SaO}_{2}$ fell below $90 \%$ and was re-inserted after $\mathrm{SaO}_{2}$ recovery.

The staff involved in each procedure included 1 senior pediatric intensivist, 2 senior pulmonologists, the rotating pediatric resident and a registered ICU nurse. A chest radiograph was obtained routinely after all procedures.

\section{Results}

\section{Patient Characteristics}

Of the 47 patients, 33 were males and 14 females with a ratio of 2.4:1. Age ranged from 1 day to 13 years with a mean $\pm \mathrm{SD}$ of $2.4 \pm 0.7$ years. The duration of primary diseases ranged from $4 \mathrm{~h}$ to 2 months with a median of 6 days. The primary diseases included severe pneumonia in 16 patients $(34.0 \%)$, foreign body aspiration in 14 (29.8\%) including 1 combined with congenital heart disease, 12 (25.5\%) with congenital structural airway abnormality, $4(8.5 \%)$ with trauma and/or prior operation and $1(2.1 \%)$ with Guillain-Barré syndrome as shown in table 1. Moreover, dyspnea and respiratory failure were noted in 36 patients (76.6\%), including 13 (27.7\%) of type I and $23(48.9 \%)$ of type II.

\section{Indication of Bronchoscopy and Bronchoscope Insertion}

Of 53 procedures, 29 (54.7\%) were performed on patients who were undergoing mechanical ventilation. Among these procedures, 38 (71.7\%) were for diagnostic reasons (upper- and lower-airway inspection, pathogen isolation), 9 (17.0\%) for therapeutic reasons (foreign body or blood clot removal, pulmonary hemorrhage, endotracheal intubation), and 6 (11.3\%) for both. The fiberoptic bronchoscope was inserted through the nasopharynx in $16(30.2 \%)$, an endotracheal tube in 33 (62.3\%), and a tracheotomy tube in $4(7.5 \%)$, as shown in table 2 .

\section{Bronchoscopic Findings}

The most common finding of fiberoptic bronchoscopy was inflammation, which was noted in $26(55.3 \%)$ patients. In these 26 patients, mucous congestion and edema without sputum plugging were found in $7(14.9 \%)$ and purulent infection with excessive secretion was noted in 19 (40.4\%), of whom 9 had mucus plugging. The second most common finding was airway foreign body aspiration, which was noted in $14(29.8 \%)$ patients: 5 (35.7\%) peanut, $4(28.6 \%)$ sunflower seeds, 2 (14.3\%) fruit, 2 (14.3\%) fruit jelly and 1 of unknown mass. The third most common finding was congenital structural airway abnormality which was noted in $12(25.5 \%)$ patients, including 8 with respiratory tract malacia (1 tracheomalacia, 2 bronchomalacia, 1 laryngotracheomalacia, 1 laryngobronchomalacia, and 2 tracheobronchomalacia, and 1 laryngotracheobronchomalacia); the other 4 had dysplasia (1 tracheal stenosis, 1 bronchial stenosis, 1 right main bronchial atresia, and 1 right superior lobar bronchus atresia). Additionally, a blood clot was found in 3 patients 
(6.4\%), including 1 with foreign body aspiration and 2 following trauma.

Segmental or lobar atelectasis was noted in 23 (48.9\%) patients: 9 (39.1\%) had mucus plugging, 8 (34.8\%) foreign body aspiration, 3 (13.0\%) blood clots and $3(13.0 \%)$ congenital airway abnormalities. BAL demonstrated pathogens in 7 (36.8\%) of 19 patients. Bacteria/yeasts were isolated in 5 procedures, which included 2 with Klebsiella pneumoniae, 1 with Pseudomonas aeruginosa, 1 with Escherichia coli, and 1 with Candida albicans. Cytomegalic inclusions and Chlamydia trachomatis were noted in 1 patient each, as shown in table 2 .

\section{Clinical Processes}

In 14 patients with foreign body aspiration, the foreign bodies were removed successfully in $11(78.6 \%)$ by fiberoptic bronchoscopy and the remaining 3 by rigid bronchoscopy in the Otolaryngology Department. In 23 patients with atelectasis, full re-expansion was obtained in $14(60.9 \%)$ patients partial re-expansion in $6(26.1 \%)$, and no change in $3(13.0 \%)$ within $24 \mathrm{~h}$ after the fiberoptic bronchoscopy procedure.

The clinical symptoms and signs of the 9 patients with mucus plugging or blood clots were significantly improved after BAL. Positive or negative microbiologic BAL results led to a modification of the treatment in 11 patients, followed by a marked clinical improvement in 9 . In this series, $13(13 / 29,44.8 \%)$ patients including 8 with foreign bodies and 5 with mucus plugging or blood clots, were extubated successfully within the following $24 \mathrm{~h}$. However, 5 (10.6\%) patients failed to improve. One remained comatose despite foreign body removal: 1 with laryngotracheobronchomalacia failed extubation, and one with pneumonia deteriorated due to pulmonary hemorrhage. Moreover, 1 patient with lower tracheal stenosis also died from pulmonary sepsis. Another patient with congenital heart disease, who had a foreign body for 2 weeks, died due to a combination of multiple organ failure and irreversible ventricular tachycardia.

\section{Complications}

Mild hypoxemia $\left(\mathrm{SaO}_{2}<90 \%\right)$ occurred transiently during $11(20.8 \%)$ procedures. Other complications included mild pulmonary hemorrhage in $2(3.8 \%)$, supraventricular tachycardia in $1(1.9 \%)$, pneumothorax in $1(1.9 \%)$, and bronchospasm in 1 (1.9\%). In patients under ventilatory support, no significant changes in ventilatory pressures were observed during the procedures. No significant airway trauma, serious hemorrhage, respiratory or cardiac arrest, or death occurred during any of these procedures.
Table 2. Bronchoscopic procedures and findings

\begin{tabular}{lc}
\hline & Patients \\
\hline Indication & \\
$\quad$ Diagnostic & $38(71.7 \%)$ \\
Therapeutic & $9(17.0 \%)$ \\
Both & $6(11.3 \%)$ \\
Bronchoscope insertion & \\
Nasopharyngeal & $16(30.2 \%)$ \\
Endotracheal tube & $33(62.3 \%)$ \\
Tracheotomy tube & $4(7.5 \%)$ \\
Bronchoscopic findings & \\
Inflammation & $26(55.3 \%)$ \\
Mucus plugging & $9(19.1 \%)$ \\
Foreign body & $14(29.8 \%)$ \\
Congenital abnormality & $12(25.5 \%)$ \\
Blood clotting & $3(6.4 \%)$ \\
BAL positive for microbes & $7 / 19(36.8 \%)$ \\
\hline
\end{tabular}

\section{Discussion}

The present report analyzes the application and value of fiberoptic bronchoscopy in a group of neonates and children admitted to our NICU and PICU. Previously, fiberoptic bronchoscopy was not encouraged in severe respiratory disorders due to concerns regarding an associated decrease in $\mathrm{PaO}_{2}$, which might cause respiratory or cardiac arrest $[13,14]$. In this work, some of our patients were in critical conditions that made them more susceptible to complications (e.g. trauma, airway foreign body, or congenital structural airway abnormality); however, their complications as well as transient lower $\mathrm{SaO}_{2}$ rates were similar to those observed in other pediatric pulmonary services $[15,16]$ where rigid or fiberoptic bronchoscopy was performed. Moreover, these complications subsided rapidly, no one died directly due to the procedure or from a complication of these procedures, although 5 cases were unimproved due to their primary disease. This suggests that fiberoptic bronchoscopy in the NICU and PICU is safe if performed by expert operators, life monitors and emergency equipment.

In this study, we noted that most of the children who underwent fiberoptic bronchoscopy were under 3 years of age. In this population (infants and toddlers), there is a higher risk for aspiration of foreign bodies, congenital airway structural abnormalities [12, 17-19] as well as weakness of cough that might predispose them to mucus plugging. Moreover, congenital airway structural abnormalities and foreign body aspiration are more prevalent 
in males $[12,17,18]$ and this correlates with our data. In this work, most procedures (71.7\%) were performed for diagnostic reasons although therapeutic management was undertaken in most patients with foreign body aspiration and airway plugging. The etiologies of 38 patients were identified by fiber-optic bronchoscopy, which is consistent with previous reports [10, 20, 21]. Moreover, therapy by fiberoptic bronchoscopy was performed after evolution by flexible bronchoscopy.

We found that mucus plugging, airway foreign body aspiration and congenital airway abnormality were the most common indications for bronchoscopy in the NICU and PICU. Of them, mucus plugging was the most common cause requiring bronchoscopy in our ICU, which might be associated with weakness of cough, serious pulmonary infection with excessive secretion, nervous system and/or muscle disorder, muscle relaxant, sedatives or anesthetic use in postoperative or mechanical ventilation. Foreign body aspiration was the second most common cause in these series. Unlike foreign body aspiration in adults, the diagnosis of foreign body aspiration in a pediatric patient is more difficult since they most commonly occur in infants and toddlers who are unable to readily communicate the circumstances leading to the aspiration event [12]. In this study, only 5 patients had an accurate history of foreign body aspiration, and most of them were unable to provide a detailed history. Congenital abnormality was another common cause that was very difficult to diagnose by history, physical examination and imaging. Bronchoscopy may be the only method for diagnosis. Furthermore, bronchoscopy can also differentiate the type and identify the severity of the abnormality [22-24]. Of 47 patients, only 2 were considered to have an airway abnormality by imaging (right pulmonary hypoplasia and narrowing of the distal trachea) that was later confirmed by bronchoscopy. It should be emphasized that general anesthesia might depress spontaneous breathing and cough which might alter the collapse of the respiratory tract wall. So, if respiratory tract malacia cannot be excluded, topical anesthesia, but not general anesthesia, should be applied in fiberoptic bronchoscopy. From these observations, we conclude that fiberoptic bronchoscopy should be performed promptly and should be helpful for diagnosis and therapy in children with foreign body aspiration, atelectasis, acute respiratory failure or respiratory tract obstruction without definite etiology.

Moreover, fiberoptic bronchoscopy is perhaps the most effective method for evaluation and diagnosis of an airway foreign body [12]. Therapeutic procedures, in- cluding foreign body and or plug removal were performed in patients with large amounts of secretions and patients with foreign body aspiration. In this study, $78.6 \%$ foreign bodies were removed by fiberoptic bronchoscopy. About $60.8 \%$ of the patients with lung atelectasis had a full reexpansion within $24 \mathrm{~h}$ after fiberoptic bronchoscopy and lavage. Eight patients with atelectasis caused by foreign bodies were extubated within $24 \mathrm{~h}$ after fiberoptic bronchoscopy. Some studies have shown that bronchoscopic suction is easy, quick, and highly effective and does not provoke any epithelial changes $[25,26]$. In children with airway inflammation, especially with mucus plugging, removal of mucus plugging can relieve the obstruction and the toxic symptoms. In this work, we also found that 5 out of 12 patients with mucus plugging and blood clotting significantly improved after bronchoscopy. Microbiological detection by BAL was very helpful for therapy, i.e. selection of the antibiotic and duration of administration. Negative microbiologic BAL findings were also important as they accelerated the search for other foci of infection and led to cessation or to downgrading of antibiotic treatment. The $44.8 \%$ rate of successful extubation within $24 \mathrm{~h}$ following fiberoptic bronchoscopy and BAL in patients with atelectasis highlights the importance of targeted, direct-vision removal of airway plugging in critically ill infants and children.

\section{Conclusion}

Our data showed that fiberoptic bronchoscopy is safe and effective in the diagnosis and therapy of serious pulmonary disorders in the NICU and PICU. We suggest that bronchoscopy should be recommended promptly, especially in patients receiving ventilation.

\section{Acknowledgements}

We thank Cai Fu Wang, Xin Er Bao, Guo Hong Zhu, Mei Ping $\mathrm{Lu}$ and Li Ping Shi for patient care arrangement. This work was partly supported by Zhejiang Science and Technology grants (2003C33007). 


\section{References}

- 1 Haas JP, Mendonca EA, Ross B, Friedman C, Larson E: Use of computerized surveillance to detect nosocomial pneumonia in neonatal intensive care unit patients. Am J Infect Control 2005;33:439-443.

$\checkmark 2$ Nayeri F, Movaghar-Nezhad K, Assar-Zadegan F: Effects of antenatal steroids on the incidence and severity of respiratory distress syndrome in an Iranian hospital. East Mediterr Health J 2005;11:716-722.

$>3$ Bratton SL, Odetola FO, McCollegan J, Cabana MD, Levy FH, Keenan HT: Regional variation in ICU care for pediatric patients with asthma. J Pediatr 2005;147:355-361.

$\checkmark 4$ Azoulay E, Schlemmer B: Diagnostic strategy in cancer patients with acute respiratory failure. Intensive Care Med 2006;32:808822 .

$\checkmark 5$ Antonelli M, Pennisi MA, Conti G, Bello G, Maggiore SM, Michetti V, Cavaliere F, Proietti R: Fiberoptic bronchoscopy during noninvasive positive pressure ventilation delivered by helmet. Intensive Care Med 2003; 29:126-129.

$\checkmark 6$ Ferrer R, Ioanas M, Agusti C, Torres A: Impact of BAL on the diagnosis and treatment of nosocomial pneumonia in ICU patients. Monaldi Arch Chest Dis 2001;56:521-526.

$>7$ Dupree HJ, Lewejohann JC, Gleiss J, Muhl E, Bruch HP: Fiberoptic bronchoscopy of intubated patients with life-threatening hemoptysis. World J Surg 2001;25:104-107.

$>8$ Silver MR, Balk RA: Bronchoscopic procedures in the intensive care unit. Crit Care Clin 1995;11:97-109.
-9 Nakano T, Shikada M, Nomura M, Kuwayama-Komaki F, Suganuma E, Ishikawa-Kato M, Sakai T, Hirakawa H, Ueno S, Yokoyama S, Niimura F, Oh Y: Feasibility of fiberoptic bronchoscopy for small infants including newborns. Tokai J Exp Clin Med 2004;29: $1-5$.

10 Bar-Zohar D, Sivan Y: The yield of flexible fiberoptic bronchoscopy in pediatric intensive care patients. Chest 2004;126:13521359.

11 Myer CM 3rd, Thompson RF: Flexible fiberoptic bronchoscopy in the neonatal intensive care unit. Int J Pediatr Otorhinolaryngol 1988;15:143-147.

12 Tang FL, Chen MZ, Du ZL, Zou CC, Zhao ZY: Fibrobronchoscopic treatment of foreign body aspiration in children: an experience of 5 years in Hangzhou City, China. J Pediatr Surg 2006;41:e1-e5.

13 Pereira W Jr, Kovnat DM, Snider GL: A prospective cooperative study of complications following flexible fiberoptic bronchoscopy. Chest 1978;73:813-816.

14 Kosloske AM: Bronchoscopic extraction of aspirated foreign bodies in children. Am J Dis Child 1982;136:924-927.

15 Zaytoun GM, Rouadi PW, Baki DH: Endoscopic management of foreign bodies in the tracheobronchial tree: predictive factors for complications. Otolaryngol Head Neck Surg 2000;123:311-316.

16 Tang LF, Xu YC, Wang CF, Zhu GH, Lu MP, Chen LX, Chen ZM: Application of flexible bronchoscopy in pediatric airway foreign body removal: experience with 1027 children from 2000-2008. World J Pediatr 2008; in press.

17 Tang LF, Du LZ, Chen ZM, Zou CC: The extracellular matrix remodeling in children with airway foreign body aspiration. Pediatr Pulmonol 2004;38:140-145.
-18 van Veenendaal MB, Liem KD, Marres HA: Congenital absence of the trachea. Eur J Pediatr 2000;159:8-13.

19 Laberge JM, Bratu I, Flageole H: The management of asymptomatic congenital lung malformations. Paediatr Respir Rev 2004; 5(suppl A):S305-S312.

20 Fan LL, Sparks LM, Fix FJ: Flexible fiberoptic endoscopy for airway problems in a pediatric intensive care unit. Chest 1988;93:556-560.

-21 Kreider ME, Lipson DA: Bronchoscopy for atelectasis in the ICU: a case report and review of the literature. Chest 2003;124:344350 .

22 Aslan AT, Kiper N, Dogru D, Karagoz AH, Ozcelik U, Yalcin E: Diagnostic value of flexible bronchoscopy in children with persistent and recurrent wheezing. Allergy Asthma Proc 2005;26:483-486.

-23 Morikawa N, Kuroda T, Honna T, Kitano Y, Fuchimoto Y, Terawaki K, Kawasaki K, Koinuma G, Matsuoka K, Saeki M: Congenital bronchial atresia in infants and children. J Pediatr Surg 2005;40:1822-1826.

24 Munro HM, Sorbello AM, Nykanen DG: Severe stenosis of a long tracheal segment, with agenesis of the right lung and left pulmonary arterial sling. Cardiol Young 2006;16:8991.

25 Sole-Violan J, Rodriguez de Castro F, Rey A, Martin-Gonzalez JC, Cabrera-Navarro P: Usefulness of microscopic examination of intracellular organisms in lavage fluid in ventilator-associated pneumonia. Chest 1994;106:889-894.

26 Coppolo DP, Brienza LT, Pratt DS, May JJ: A role for the respiratory therapist in flexible fiberoptic bronchoscopy. Respir Care 1985; 30:323-327. 